\title{
O treinamento físico como estratégia de intervenção em pessoas com Insuficiência Renal Crônica
}

\author{
Physical training as an intervention strategy in people with Chronic Kidney Insufficiency \\ El entrenamiento físico como estrategia de intervención en personas con Insuficiencia \\ Renal Crónica
}

Cássio Murilo Almeida Lima Júnior ${ }^{1 *}$, Wilson Pereira Lima² ${ }^{2}$ Lúcio Marques Vieira Souza ${ }^{3}$, Claudia Mara Santos Souza ${ }^{4}$, Jorge Rollemberg dos Santos ${ }^{5}$, Frederico Barros Costa ${ }^{1}$, André Luiz de Santana Silva ${ }^{6}$, Evelini Veras de Jesus ${ }^{1}$, Paula Esteves Carvalho ${ }^{1}$, Estélio Henrique Martin Dantas $^{1,7}$.

\section{RESUMO}

Objetivo: Identificar os indicadores positivos e negativos do treinamento físico em pessoas com Insuficiência Renal Crônica (IRC), para subsidiar os profissionais e acadêmicos de educação física e áreas afins, como referencial teórico do treinamento físico para nefropatas. Revisão bibliográfica: A pessoa com IRC possui uma grande perda de massa muscular, que é o mais significante indicador de mortalidade nas pessoas em tratamento de hemodiálise. A perda de massa muscular, sarcopenia, pode ser um risco mitigado pela atividade física, que exerce um controle importante na reversão dessa perda, ressaltando que os programas de exercícios físicos aeróbios e/ou de força, quando adequadamente prescritos durante a hemodiálise, são seguros para os pacientes e podem gerar uma gama de benefícios para estes indivíduos. Considerações finais: O estudo aponta que treinamento físico apresenta benefícios para a melhora do estado geral e da qualidade de vida dos pacientes renais crônicos, sendo observada melhorias em diversas funções, tendo influência direta na qualidade de vida desse grupo, podendo ser adotado como proposta não farmacológicas seguras e eficazes para pessoas com IRC.

Palavras-chave: Insuficiência Renal Crônica, Exercício físico, Qualidade de vida.

\begin{abstract}
Objective: To identify the positive and negative indicators of physical training in people with Chronic Renal Insufficiency (CRF), to support professionals and academics of physical education and related areas, as a theoretical reference for physical training for nephropaths. Bibliographic review: The person with CRF has a great loss of muscle mass, which is the most significant indicator of mortality in people undergoing hemodialysis. The loss of muscle mass, sarcopenia, can be a risk mitigated by physical activity, which exerts an important control in reversing this loss, emphasizing that aerobic and / or strength exercise programs, when properly prescribed during hemodialysis, are safe for patients and can generate a range of benefits for these individuals. Final considerations: The study points out that physical training has benefits for the improvement of the general condition and quality of life of chronic kidney patients, with improvements in several functions being observed, having a direct influence on the quality of life of this group, which can be adopted as a nonpharmacological proposal safe and effective for people with CKD.
\end{abstract}

Keywords: Chronic Kidney Diseases, Physical exercise, Quality of life.

1 Universidade Tiradentes (UNIT), Aracaju - SE. *E-mail: murilo.cassio@hotmail.com

2 Universidade Municipal de São Caetano do Sul (USCS), São Caetano do Sul - SP.

3 Universidade do Estado de Minas Gerais (UEMG), Passos - MG.

${ }^{4}$ Universidade Federal de Sergipe (UFS), Lagarto - SE.

${ }^{5}$ Universidade Federal de Sergipe (UFS), São Cristóvão - SE.

${ }^{6}$ Faculdade de Desporto da Universidade do Porto (FADEUP), Porto - Portugal.

7 Universidade Federal do Estado do Rio de Janeiro (UNIRIO), Rio de Janeiro - RJ. 


\section{RESUMEN}

Objetivo: Identificar los indicadores positivos y negativos del entrenamiento físico en personas con Insuficiencia Renal Crónica (IRC), para apoyar a los profesionales y académicos de la educación física y áreas afines, como referente teórico para el entrenamiento físico de nefrópatas. Revisión bibliográfica: La persona con IRC tiene una gran pérdida de masa muscular, que es el indicador más significativo de mortalidad en personas en hemodiálisis. La pérdida de masa muscular, sarcopenia, puede ser un riesgo mitigado por la actividad física, la cual ejerce un control importante en la reversión de esta pérdida, enfatizando que los programas de ejercicios físicos aeróbicos y / o de fuerza, cuando se prescriben adecuadamente durante la hemodiálisis, son seguros. para los pacientes y puede generar una serie de beneficios para estas personas. Consideraciones finales: El estudio señala que el entrenamiento físico tiene beneficios para la mejora del estado general y la calidad de vida de los pacientes renales crónicos, observándose mejoras en varias funciones, incidiendo directamente en la calidad de vida de este grupo, lo que puede ser adoptado como una propuesta no farmacológica seguro y eficaz para personas con ERC.

Palabras clave: Insuficiencia Renal Crónica, Ejercicio físico, Calidad de vida.

\section{INTRODUÇÃO}

A Insuficiência Renal Crônica (IRC) é a perda progressiva e irreversível da função renal, urinária e endócrina, na qual o organismo não mantém o equilíbrio metabólico e hidroeletrolítico, finalizando em um quadro urêmico, síndrome clínica em que compromete o funcionamento de diversos sistemas ou órgãos (DOUGLAS CR, 2001).

Dessa forma, a IRC caracteriza-se como uma condição em que os rins são acometidos por um déficit funcional progressivo por mais de três meses, e é classificada em cinco estágios de acordo com a redução da Taxa de Filtração Glomerular (TFG) e do grau de lesão dos rins, medido pelo nível de proteinúria (MEDICAL SUBJECT HEADINGS, 2006). As funções bioquímicas e fisiológicas são afetadas devido ao acúmulo de metabólitos e desequilíbrio hidroeletrolítico, hipovolemia, entre outros fatores (FAGUNDES RAB, et al., 2018). Devido ao aumento da população idosa e de fatores de risco como hipertensão, diabetes, e doenças cardiovasculares, a IRC é considerada um dos maiores desafios da Saúde Pública no século atual (SILVA PAB, et al., 2020).

O tratamento da IRC dependerá a princípio de seu estágio, ou quadro. Observa-se que as práticas terapêuticas mais comuns envolvem os seguintes fatores: 1- Combate a fatores de risco (Hipertensão Arterial, Diabetes, Dislipidemia); 2- Alterações alimentares; 3- Redução da massa corporal; e 4- Abstinência do fumo; estratégias que exercem efeitos mais significativos em estágios iniciais da IRC (MAGALHÃES GF e GOULART RMM, 2015). Intervenções complementares, através da acupuntura auricular, eletroacupuntura e acupressão, podem colaborar com tratamentos convencionais através da redução de sintomas agudos como insônia, dor, náuseas, fadiga, ansiedade, entre outros (MELO GAA, et al., 2020).

Já em estágios avançados o tratamento pode envolver terapia renal substitutiva por meio de hemodiálise, diálise peritoneal ambulatória contínua, diálise peritoneal cíclica, e diálise peritoneal intermitente, além do transplante, a IRC é um grande problema de saúde, sendo exposta como uma doença complexa que exige múltiplas abordagens no seu tratamento, mostrando que não é incomum pessoas com IRC serem encaminhadas a tratamento nefrológico, quando já estão em fase avançada da doença necessitando de terapia dialítica de urgência ou emergência (BASTOS MG e KIRSZTAJN GM, 2011).

Com a debilitação do organismo, a IRC provoca alterações físicas associadas ao tratamento, constituindo fatores limitantes das atividades diárias, uma vez que os pacientes ficam impossibilitados de realizarem por exemplo passeios e viagens prolongadas e como consequência a diminuição da atividade física, a prática de esportes e as atividades socais, principalmente na presença de sintomas como anemia, que induz intolerância aos exercícios (BASTOS MG e KIRSZTAJN GM, 2011; RIBEIRO R, et al., 2013).

O tratamento hemodialítico é responsável por um cotidiano monótono e restrito, e as atividades desses indivíduos são limitadas após o início do tratamento, favorecendo o sedentarismo, a deficiência funcional, 
baixo fator emocional e baixa vitalidade, as pessoas com IRC sofrem alterações da vida diária, por conta do tratamento que necessita de um suporte grande, a pessoa fica dependente da equipe de saúde, do equipamento para hemodiálise, isso tudo são fatores que refletem na qualidade de vida dessas pessoas (MARTINS MRI e CESARIANO CB, 2005). Fato este constatado ao serem evidenciadas baixas pontuações com relação à função física e emocional após verificação do nível de qualidade de vida de indivíduos com IRC (CORDEIRO EDDO, et al., 2020).

No entanto, o treinamento físico pode ser compreendido como um regime planejado e estruturado de exercício físicos regulares, realizados deliberadamente para melhorar a aptidão física, estando o exercício físico relacionado com a capacidade de realizar tarefas diárias com vigor e atenção, sem fadiga excessiva e com ampla energia para desfrutar de atividades de lazer e para atender a emergências imprevistas, sendo uma intervenção potencialmente facilmente acessível e econômica, que pode ser fornecida por meio de grupos de exercícios ou profissionais da educação física trabalhando em conjunto com profissionais de saúde (BARTELS B, et al., 2019).

Então o exercício físico pode ser considerado como uma importante intervenção terapêutica e que poderá melhorar as alterações fisiológicas, psicológicas e funcionais no tratamento desses indivíduos (MARCHESAN M e ROMBALDI AJ, 2012; NASCIMENTO LCDA, et al., 2012; RIBEIRO R, et al., 2013). Apesar desses fatores, percebe-se a escassez de intervenções e programas voltadas para essa população, especialmente para idosos com IRC, devido às suas maiores limitações (MARCHESAN M, et al., 2016).

Nos pacientes quando submetidos ao treinamento físico são observadas melhorias em diversas funções, tais como como a pressão arterial, função ventricular, melhoria da força muscular, capacidade respiratória, redução da atrofia muscular e da qualidade de vida (KOH KP, et al., 2009; SMART N e STEELE M, 2011).

Portanto, o presente estudo tem como objetivo realizar uma revisão narrativa da literatura sobre o treinamento físico como estratégia de intervenção em pacientes com insuficiência renal crônica para subsidiar os profissionais e acadêmicos de educação física e áreas afins, como referencial teórico do treinamento físico para nefropatas.

\section{REVISÃO BIBLIOGRÁFICA}

\section{Insuficiência renal crônica}

Os rins são órgãos que lembram a forma de um grão de feijão, de coloração marrom-avermelhada, situados no espaço retroperitoneal, um de cada lado da coluna vertebral, de tal forma que seu eixo longitudinal corre paralelamente ao músculo psoas maior (RIELLA MC, 2014).

Geralmente, o rim filtra o sangue e o depura de produtos do metabolismo proteico, enquanto previne a perda de solutos específicos, proteínas (particularmente a albumina) e os componentes celulares encontrados no sangue (BASTOS MG, et al., 2011). Além disso os rins executam outras funções importantes, como regulação da água do organismo e outros elementos químicos do sangue como o sódio, o potássio, o fósforo e o cálcio; eliminam medicamentos e toxinas introduzidos no organismo; liberam hormônios no sangue, hormônios que fabricam células vermelhas do sangue, que fortalecem os ossos e regulam a pressão arterial (SOCIEDADE BRASILEIRA DE NEFROLOGIA, 2015).

Quando há uma disfunção na função dos rins acontece a IRC, que é a condição na qual os rins perdem a capacidade de efetuar suas funções básicas, sendo uma perda lenta, progressiva e irreversível, sendo que grande parte das pessoas não apresentam sintomas graves até que a IRC esteja em um estágio avançando, entretanto é possível observar alguns sintomas como: cansaço, falta de energia, dificuldade de concentração, falta de apetite, dificuldades para dormir, cãibras noturnas, pés e tornozelos inchados, pele seca e irritada, inchaço ao redor dos olhos principalmente no período da manhã e o aumento da frequência da urina no período noturno (MINISTÉRIO DA SAÚDE, 2019). Comorbidades como o diabetes e a hipertensão são fatores de risco para o desenvolvimento da IRC, e um dos principais gatilhos para esses distúrbios é a obesidade (SOCIEDADE BRASILEIRA DE NEFROLOGIA, 2018). 
Das diversas formas existentes em detectar as doenças renais crônicas, estão em destaque um exame de urina e os exames detalhados dos rins, como a dosagem de marcadores indiretos como a creatinina, sendo que a função excretora é a que tem maior correlação com os resultados clínicos. Com a redução da função excretora, ocorre paralelamente o declínio da função renal. A função excretora renal pode ser avaliada pela Taxa de Filtração Glomerular (TFG). São utilizadas as seguintes referências para um diagnóstico de uma pessoa com doença renal crônica: TFG alterada, TFG normal ou próxima do normal, sendo que qualquer pessoa, que manifeste pelo menos por três meses contínuos uma $T F G<60 \mathrm{ml} / \mathrm{min} / 1,73 \mathrm{~m}^{2}$, diagnosticada com doença renal crônica (MINISTÉRIO DA SAÚDE, 2019).

A doença renal cônica está dividida em seis estágios, que funcionam de acordo com o grau de função renal, o estágio inicial é o zero, onde se encontram os grupos de riscos com ausência de leão renal, até o estágio mais avançado que é a doença renal crônica terminal, nessa fase as opções de tratamento são os métodos de terapia substitutivas ou o transplante renal (JUNIOR JER, 2004).

De acordo com a sociedade brasileira de nefrologia (2018), encontra-se sob tratamento dialítico no Brasil uma estimativa de 126.583 pacientes e a fila de espera para transplante teve alta de 29.268 pacientes (2016) para os atuais 31.266. Na IRC o tratamento hemodialítico, provoca uma sucessão de situações para o paciente renal crônico que compromete o aspecto não só físico, como psicológico, com repercussões pessoas, familiares e sociais, sendo que esta mudança subitânea de vida vem acompanhada com o temor da morte, mas também vem seguido com a expectativa de um transplante renal, junto com a esperança de melhorar a sua qualidade de vida (CESARINO C.B e CASAGRANDE LDR, 1998).

Diante das complicações cardiorrespiratórias às quais pacientes nefropatas crônicos podem estar submetidos, o exercício físico, bem como diferentes intervenções reabilitativas, tem demonstrado grande relevância clínica (CARVALHO TD, 2006). Entretanto, ainda não foi notada como rotina na prática clínica a aplicação efetiva de programas de exercícios para doentes renais crônicos como terapia não medicamentosa (MARTINS MRI e CESARINO CB, 2004). Observam-se controvérsias acerca de aspectos ambientais e metodológicos relacionados ao treinamento, como volume, intensidade ou local (NAJAS et al., 2009).

\section{Treinamento físico e insuficiência renal crônica}

O treinamento físico pode ser compreendido como um processo organizado e sistemático de aperfeiçoamento físico, nos seus aspectos morfológicos e funcionais, impactando diretamente sobre a capacidade de execução de tarefas que envolvam demandas motoras, sejam elas esportivas ou não, sendo que o comportamento físico-esportivo de cada pessoa é consequência da interação de diversos fatores, entre eles os fisiológicos, biomecânicos e psicológicos. (ROSCHEL H, et al., 2011).

Um estudo alcançado por Peres CPA, et al. (2009) realizado em pessoas com doença renal crônica, submetidas a um programa de treinamento físico com uma frequência de 3 vezes por semana, sendo 1 hora por dia, realizados ao longo das sessões de hemodiálise, contando com a presença de cinquenta e oito pacientes, no programa de treinamento físico foram utilizados equipamentos de custo razoável, baratos, de fácil acesso, foram utilizados o teste de caminhada de seis minutos; o shuttle teste, para aferir o consumo máximo de oxigênio; o teste de 1 repetição máxima, para aferir a força muscular do quadríceps; o teste de pressão respiratória máxima; a espirometria e a qualidade de vida de vida avaliados pelo Medical Outcomes Study Short-Form 36. O autor concluiu que em um programa de 2 meses de treinamento com exercícios, demostrou ser satisfatório para ocorrer um aumento expressivo da capacidade física, tendo como consequência o aumento da qualidade de vida desses pacientes.

Em outro estudo realizado por De Lima FF, et al. (2013), foram avaliados os efeitos de um programa de exercício físico sobre a função pulmonar, capacidade funcional, qualidade de vida e dor, em pacientes que realizam hemodiálise. Participaram 28 pacientes de ambos os sexos com idade entre 40 e 60 anos em programa de hemodiálise. A força muscular respiratória foi avaliada pela manovacuometria, a capacidade funcional pelo TC6', a qualidade de vida pelo questionário KDQOLSF, a função pulmonar pela espirometria e a dor pela EVA. Após as avaliações, os pacientes iniciaram o programa de exercícios 3 vezes por semana, 40 minutos durante a hemodiálise, no período de 8 semanas. Após os resultados teve a conclusão que embora 
a capacidade pulmonar e a capacidade funcional (TC6') não tenham apresentado alterações ao final do experimento, os níveis reduzidos de dor, cansaço e dispneia sugerem melhora do desempenho funcional, após programas de exercício físico.

No estudo de Dantas FF e Figueirôa NMC (2014) foi analisado a influência do treinamento aeróbio intradialítico em parâmetros fisiológicos, bioquímicos e funcionais, em um grupo de pacientes renais crônicos, durante 12 semanas de exercícios realizados num cicloergômetro, três dias por semana, numa intensidade correspondente a $50 \%$ - $60 \%$ da frequência cardíaca de reserva e apresentou como conclusão que foi capaz de proporcionar melhoras em variáveis fisiológicas, bioquímicas e funcionais nos pacientes submetidos à hemodiálise. Em um estudo similar, Coelho DM et al. (2007), porém com base no limiar de lactato em paciente com IRC submetido à hemodiálise, e o protocolo consistindo em 3 sessões semanais durante 8 semanas, verificou uma melhora da capacidade funcional e na resposta do metabolismo oxidativo.

Utilizando o Teste de Caminha de 6 minutos (TC6M), Corrêa LB, et al. (2009) utilizou um programa de treinamento muscular duas vezes por semana durante cinco meses aplicados em pacientes com idade entre 29 e 84, portadores de doença renal crônica, e verificou que durante a sessão de hemodiálise o treinamento foi capaz de promover o aumento da força muscular dos membros inferiores, além de alterar positivamente a qualidade de vida, e redução da dor nos membros inferiores e das câimbras.

Já no estudo de Ribeiro $R$, et al. (2013) com um programa de exercício resistido no tratamento e na qualidade de vida em pacientes submetidos à hemodiálise crônica, submetendo pacientes com doença renal crônica e diabetes mellitus ao exercício resistido durante as sessões de hemodiálise durante 8 semanas e 3 vezes por semana, teve como resultados melhoras em variáveis bioquímicas, parâmetros clínicos e na força muscular no período intradialítico.

Soares KTDA, et al. (2011) analisou os efeitos de um protocolo de exercícios fisioterapêuticos em pacientes renais crônicos, durante a terapia hemodialítica, foram avaliados 27 pacientes, com idade média de 51 anos. Durante três meses, participaram de um programa de tratamento, durante a hemodiálise, composto por alongamentos musculares de membros inferiores, de membro superior, lombar e cervical; fortalecimentos musculares de membros inferiores e membro superior, sem o acesso vascular; e relaxamento. O questionário "Medical Outcomes Study 36 (SF36)", foi aplicado antes e após o período de tratamento. Após o tratamento foi observado melhoras na capacidade funcional, nível de dor, vitalidade e saúde mental. E concluiu que os programas de reabilitação física são benéficos para a melhora do estado geral e da qualidade de vida dos pacientes renais crônicos.

Henrique DMN, et al. (2010) realizou um estudo em 14 pacientes com média de 48 anos e identificou que o treinamento aeróbico durante as sessões de hemodiálise repercutia sobre a capacidade funcional, assim como sobre a pressão arterial dos pacientes renais crônicos desse tipo de terapia substitutiva renal. Para avaliação da capacidade física, realizou-se o teste de caminhada de 6 minutos (TC6M) e o teste cardiopulmonar de exercício (TCPE), em um período de 12 semanas. Concluiu-se que a partir disso foi possível concluir que havia um impacto de melhora da capacidade funcional e do controle da pressão arterial dos pacientes dialíticos submetidos ao treinamento aeróbico durante a sessão de hemodiálise sob essa metodologia.

Marchesan M e Rombaldi AJ (2012) apresentaram um programa de exercícios físicos para pacientes em hemodiálise que foi desenvolvido na Clínica Renal do Hospital Santa Lúcia, (Cruz Alta-RS), com 72 pacientes na unidade de hemodiálise, os exercícios foram desenvolvidos durante as sessões de hemodiálise, executados na seguinte sequência: alongamento antes de iniciar as sessões de hemodiálise; aquecimento realizado na bicicleta estacionária sem carga com duração de 3 minutos; treinamento aeróbio na bicicleta estacionária com duração inicial de 15 minutos; exercícios de resistência muscular localizada. Conclui-se programa de exercícios físicos trouxe benefícios para a reabilitação dos pacientes uma vez que contribuiu com a melhora da aptidão física e da qualidade de vida dos mesmos. Esses ganhos foram identificados no desempenho nos testes físicos, bem como nos relatos das entrevistas, onde os pacientes puderam se posicionar com relação à participação no programa.

Na realização de um estudo cujo o objetivo foi determinar os efeitos do exercício físico em pacientes no processo de hemodiálise, durante um período de 5 meses em 33 pacientes, foram realizados teste de escada 
de 2 min, teste de agachamento, questionário "Medical Outcomes Study 36 (SF36), pressão arterial e lipídios. O exercício físico realizado duas vezes por semana, durante 5 meses, aumentou a função física e a capacidade aeróbia em pacientes em hemodiálise. Mais investigações são necessárias para determinar os efeitos sobre a pressão arterial e lipídios. Não houve complicações médicas relacionadas ao programa de exercício (MOLSTED S, et al., 2004).

A aplicação de programas de exercícios no doente renal crônico, na prática clínica, ainda não se tornou rotina (MARTINS MRI e CESARINO CB, 2004). Para Garrett WE e Kirkendall DT (2003) o treinamento não tem sido associado a uma melhora da falha renal crônica ou a uma diminuição na incidência da disfunção renal, de maneira contrária aos efeitos da atividade física nas doenças cardiovasculares. Apesar do exercício não ser comumente prescrito para esse público-alvo, verifica-se ausência de evidências que possam sugerir sua contraindicação (NAJAS et al., 2009; CHEEMA BSB, et al., 2005). A sarcopenia, caracterizada pela perda de massa muscular, apresenta relação importante com mortalidade nas pessoas em tratamento de hemodiálise, sendo o fator de maior impacto, ela pode ser causada por conta da perda de aminoácidos nas sessões de hemodiálise (CHEEMA BSB, et al., 2005).

A perda de massa muscular, sarcopenia, pode ser um risco mitigado pela atividade física, que exerce um controle importante e reversão dessa perda, ressaltando que os programas de exercícios físicos aeróbios e/ou de força, quando adequadamente prescritos durante a hemodiálise, são seguros para os pacientes e podem gerar uma gama de benefícios para estes indivíduos (COELHO DM, et al., 2008). Os programas de reabilitação para pacientes renais crônicos trazem benefícios, melhorando a qualidade de vida dessas pessoas, sendo que esta melhora pode ser considerada uma finalidade compartilhada por esta população (MARTINS MRI e CESARINO CB, 2004).

Nesse sentido, observa-se que exercícios físicos, intervenções sistematizadas e não farmacológicas, conferem benefícios cardiorrespiratórios, neuromusculares e bioquímicos para pacientes renais crônicos, traduzindo-se em melhorias hemodinâmicas, mesmo durante as fases dialíticas ou interdialíticas (NAJAS CS, et al., 2009). O exercício físico independente da modalidade, seja aeróbico, seja de resistência ou até mesmo uma combinação desses, apresenta efeitos incrementais na capacidade funcional, na força muscular e na qualidade de vida dos pacientes com IRC, portanto, deve-se levar em consideração o treinamento físico como uma modalidade terapêutica relevante (NASCIMENTO LCDA, et al., 2012).

\section{CONSIDERAÇÕES FINAIS}

É notável que o treinamento físico apresenta benefícios para a melhora do estado geral e da qualidade de vida dos pacientes renais crônicos, sendo observada melhorias em diversas funções, tais como como a pressão arterial, função ventricular, melhoria da força muscular, capacidade respiratória, redução da atrofia muscular e da qualidade de vida. O treinamento físico pode ser adotado como proposta não farmacológicas seguras e eficazes para pessoas com IRC. Sugere-se, portanto, abrir um novo panorama e estimular ainda mais a realização de novas pesquisas nessa área, para subsidiar os profissionais que atuarão diretamente no tratamento dos pacientes.

\section{AGRADECIMENTOS E FINANCIAMENTO}

O presente trabalho foi realizado com apoio da Coordenação de Aperfeiçoamento de Pessoal de Nível Superior - Brasil (CAPES) - Código de Financiamento 001.

\section{REFERÊNCIAS}

1. BARTELS B, et al. Physical exercise training for type 3 spinal muscular atrophy. Cochrane Database of Systematic Reviews, 2019; (3).

2. BASTOS MG, KIRSZTAJN GM. Chronic kidney disease: importance of early diagnosis, immediate referral and structured interdisciplinary approach to improve outcomes in patients not yet on dialysis. Brazilian Journal of Nephrology, 2011; 33(1), 93-108.

3. BASTOS MG, et al. Doença renal crônica no paciente idoso. Clinical \& Biomedical Research, 2011; 31(1).

4. CARVALHO TD. Diretriz de reabilitação cardiopulmonar e metabólica: aspectos práticos e responsabilidades. Arquivos Brasileiros de Cardiologia, 2006; 86, 74-82. 
5. CESARINO CB, CASAGRANDE LDR. Paciente com insuficiência renal crônica em tratamento hemodialítico: atividade educativa do enfermeiro. Revista latino-americana de enfermagem, 1998; 6(4), 31-40.

6. CHEEMA BSB, et al. A rationale for intradialytic exercise training as standard clinical practice in ESRD. American Journal of Kidney Diseases, 2005; 45(5), 912-916.

7. CHEEMA BSB, SINGH M AF. Exercise training in patients receiving maintenance hemodialysis: a systematic review of clinical trials. American journal of nephrology,2005; 25(4), 352-364.

8. COELHO DM, et al. Exercícios físicos durante a hemodiálise: uma revisão sistemática. J Bras Nefrol, 2008; 30(2), 8898.

9. CORDEIRO EDDO, et al. Quality of life of individuals receiving kidney transplantation in Amazonas State. Revista Latino-Americana de Enfermagem, 2020; 28.

10. COELHO DM, et al. Avaliação funcional e prescrição de treinamento para paciente portador de insuficiência renal crônica submetido a hemodiálise: um relato de caso. RBPFEX-Revista Brasileira de Prescrição e Fisiologia do Exercício,2007; 1(3).

11. CORRÊA LB, et al. Efeito do treinamento muscular periférico na capacidade funcional e qualidade de vida nos pacientes em hemodiálise. J. Bras. Nefrol, 2009; 31(1), 18-24.

12. DANTAS FFO, FIGUEIRÔA NMC. Avaliação dos efeitos do treinamento aeróbio intradialítico em pacientes renais crônicos. Revista de Atenção à Saúde, 2014; 12(42).

13. DE LIMA FF, et al. Avaliação funcional pré e pós-programa de exercício físico de pacientes em hemodiálise. Medicina (Ribeirão Preto. Online), 2013; 46(1), 24-35.

14. DOUGLAS CR. Patofisiologia de sistemas renal. São Paulo: Robe, 2001; 62-7.

15. FAGUNDES RAB, et al. Probiotics in the treatment of chronic kidney disease: a systematic review. Brazilian Journal of Nephrology, 2018; 40(3), 278-286.

16. GARRETT WE, KIRKENDALL DT. A ciência do exercício e dos esportes. Porto Alegre: Ed. Artmed, 2003.

17. HENRIQUE DMN, et al. (2010). Treinamento aeróbico melhora a capacidade funcional de pacientes em hemodiálise crônica. Arquivos Brasileiros de Cardiologia, 2010; 94(6), 823-828.

18. JUNIOR JER. Doença renal crônica: definição, epidemiologia e classificação. J. Bras. Nefrol, 2004; 26(3 suppl. 1), 13.

19. $\mathrm{KOH} \mathrm{KP}$, et al. Intradialytic versus home based exercise training in hemodialysis patients: a randomised controlled trial. BMC nephrology, 2009; 10(1), 2.

20. MAGALHÃES FG, GOULART RMM. Doença renal crônica e tratamento em idosos: uma revisão integrativa. Revista Brasileira de Geriatria e Gerontologia, 2015; 18(3), 679-692.

21. MARCHESAN M, et al. Physical exercise modifies the functional capacity of elderly patients on hemodialysis. Fisioterapia em Movimento, 2016; 29(2), 351-359.

22. MARCHESAN M, ROMBALDI AJ. Programa de exercícios físicos para o doente renal crônico em hemodiálise. Revista Brasileira de Atividade Física \& Saúde, 2012; 17(1), 75-78.

23. MARTINS MRI, CESARINO CB. Atualização sobre programas de educação e reabilitação para pacientes renais crônicos submetidos à hemodiálise. J Bras Nefrol, 2004; 26(1), 45-50.

24. MARTINS MRI, CESARINO CB. Qualidade de vida de pessoas com doença renal crônica em tratamento hemodialítico. Revista Latino-Americana de Enfermagem,2005; 13(5), 670-676.

25. MEDICAL SUBJECT HEADINGS. 2006. Renal Insufficiency, Chronic - MeSH - NCBI. Disponível em: https://www.ncbi.nlm.nih.gov/mesh/68051436. Acesso em: 22 set. 2020.

26. MELO GAA, et al. Effects of acupuncture in patients with chronic kidney disease: a systematic review. Revista Brasileira de Enfermagem, 2020; 73(4).

27. MOLSTED S, et al. Five months of physical exercise in hemodialysis patients: effects on aerobic capacity, physical function and self-rated health. Nephron Clinical Practice, 2004; 96(3), c76-c81.

28. MINISTÉRIO DA SAÚDE. 2019. Doenças renais: causas, sintomas, diagnóstico, tratamento e prevenção. [S. I.]. Disponível em: http://www.saude.gov.br/saude-de-a-z/doencas-renais. Acesso em: 6 out. 2020.

29. NAJAS CS, et al. Segurança e eficácia do treinamento físico na insuficiência renal crônica. Revista Brasileira de Medicina do Esporte, 2009; 15(5), 384-388.

30. NASCIMENTO LCDA, et al. Efetividade do exercício físico na insuficiência renal crônica. Fisioterapia em Movimento, 2012; 25(1), 231-239.

31. PERES CPA, et al. Efeitos de um programa de exercícios físicos em pacientes com doença renal crônica terminal em hemodiálise. J. Bras. Nefrol, 2009; 31(2), 105-113.

32. ROSCHEL H, et al. Treinamento físico: considerações práticas e científicas. Revista Brasileira de Educação Física e Esporte, 2011; 25, 53-65.

33. SILVA PAB, et al. Brazilian public policy for chronic kidney disease prevention: challenges and perspectives. Revista de Saúde Pública, 2020; 54, 86.

34. RIBEIRO R, et al. Efeito do exercício resistido intradialítico em pacientes renais crônicos em hemodiálise. Brazilian Journal of Nephrology, 2013; 35(1), 13-19.

35. SMART N, STEELE M. Exercise training in haemodialysis patients: a systematic review and metaanalysis. Nephrology, 2011; 16(7), 626-632.

36. RIELLA MC. Princípios de nefrologia e distúrbios hidroeletrolíticos. 5a ed. Editora Guanabara Koogan: Rio de Janeiro, 2014.

37. SOARES KTDA, et al. Eficácia de um protocolo de exercícios físicos em pacientes com insuficiência renal crônica, durante o tratamento de hemodiálise, avaliada pelo SF-36. Fisioterapia em Movimento, 2011; 24(1), $133-140$.

38. SOCIEDADE BRASILEIRA DE NEFROLOGIA. 2015. Insuficiência renal crônica. [S. I.]. Disponível em: http://bvsms.saude.gov.br/dicas-em-saude/2083-insuficiencia-renal-cronica. Acesso em: 6 jul. 2020.

39. SOCIEDADE BRASILEIRA DE NEFROLOGIA. 2018. Fatores de risco para a Doença Renal Crônica. [S. I.]. Disponível em: https://sbn.org.br/fatores-de-risco-para-a-doenca-renal-cronica/?publico. Acesso em: 6 out. 2020.

40. SOCIEDADE BRASILEIRA DE NEFROLOGIA. 2018. Censo de diálise revela 40 mil novos pacientes em 2017 no Brasil. Rio de Janeiro, 2018. Disponível em: https://arquivos.sbn.org.br/uploads/sbninforma114-2.pdf . Acesso em: 12 out. 2020 . 